\title{
PTU-059 PREVALENCE OF OSTEOPOROSIS IN LEEDS COELIAC COHORT
}

doi:10.1136/gut.2011.239301.187

T Fitzgerald, ${ }^{1}$ H Davies, ${ }^{1}$ P D Howdle, 1 S M Everett, ${ }^{1}$ J S Jennings ${ }^{1 *}$ Department of Gastroenterology, Leeds Teaching Hospitals NHS Trust, Leeds, UK

Introduction Coeliac disease may be associated with low bone mineral density, osteoporosis, and increased fracture risk. ${ }^{1}$ We aimed to identify patients with coeliac disease and osteoporosis as defined by bone mineral density analysis.

Methods A database of patients with coeliac disease was established in Leeds in 2001 . We retrospectively analysed this database to look at individuals who had undergone a bone mineral density scan. Osteoporosis was defined as a $\mathrm{T}$ score $-2.5 \mathrm{SDs}$ below mean at either hip or lumbar spine.

Results A total of 202 individuals were recorded on the database with complete bone density data. Overall 24\% male, $76 \%$ female, mean age at diagnosis 42 years (range 0-84 years). Initial duodenal biopsy by Marsh grade: $023.5 \%$, I $7.0 \%$, II $29.0 \%$, III $23.0 \%$ and IV 17.5\%. Compliance with a Gluten Free Diet (GFD) was recorded as poor $10.4 \%$, good $57.2 \%$, excellent $1.2 \%$ and unknown $31.2 \%$. Smoking status was available for 93 individuals. 9.4\% were current smokers, $33.2 \%$ had never smoked. $24.9 \%$ consumed alcohol. $24.75 \%$ were osteoporotic on bone mineral density scan. Osteoporosis was not associated with age at diagnosis $(p=0.82)$, gender $(p=0.16$ for male vs female), compliance with GFD ( $p=0.80)$ or smoking status $(p=0.53)$. Osteoporosis was associated with any level of alcohol consumption $(p=0.04)$. Comparing Marsh grade to osteoporosis: Grade III was found in $28 \%$ and IV in $26 \%$ of those with osteoporosis. This was not statistically significant.

Conclusion Nearly $25 \%$ of patients had osteoporosis. This did not correlate with age at diagnosis, gender, smoking or dietary compliance. There was an association with alcohol. Our prevalence is higher than that found by Lewis et al $(n=43$, hip $7 \%$, spine $14 \%$ ) and would support routine screening for osteoporosis in coeliac disease. ${ }^{2}$

Competing interests None.

Keywords bone mineral density, Coeliac Disease, osteoporosis.

\section{REFERENCES}

1. Ludvigsson JF, Michaelsson K, Ekbom A, et al. Coeliac disease and the risk of fractures - a general population-based general cohort study. APT 2007;25:273-85.

2. Lewis NR, Scott BB, et al. Should patients with coeliac disease have their bone mineral density measured? Eur J Gastroenterol Hepatol 2005;17:1065-70. 